\title{
Entanglement versus energy in quantum spin models
}

\author{
XiaoGuang Wang \\ Zhejiang Institute of Modern Physics, Department of Physics, Zhejiang University, HangZhou 310027, China
}

(Dated: November 13, 2018)

\begin{abstract}
We study entanglement properties of all eigenstates of the Heisenberg $X X X$ model, and find that the entanglement and mixedness for a pair of nearest-neighbor qubits are completely determined by the corresponding eigenenergies. Specifically, the negativity of the eigenenergy implies pairwise entanglement. From the relation between entanglement and eigenenergy, we obtain finite-size behaviors of the entanglement. We also study entanglement and mixedness versus energy in the quantum Heisenberg $X Y$ model.

PACS numbers: 03.65.Ud, 75.10.Jm
\end{abstract}

Quantum entanglement lies at the heart of quantum mechanics, and can be exploited to accomplish some physical tasks such as quantum teleportation [1]. In this sense, it can be regarded as a resource, just like energy. As pointed out by Osborne and Nielsen [2], the similarity between entanglement and energy turns out to be more than superficial. It is interesting to explore the relationship between these two resources, entanglement and energy.

Recently, the study of entanglement properties in many-body systems have received much attention [8? 19]. Specifically, for the ground state (zero temperature) of a ring of $N$ qubits interacting via the antiferromagnetic isotropic Heisenberg Hamiltonian, a direct relation is established between the concurrence $C$ [20] quantifying the two-qubit entanglement and the ground-state energy per site $\epsilon_{0}[10,21,22]$ :

$$
C_{0}(N)=\max \left[0,-\mathcal{E}_{0}(N) / N\right]=\max \left[0,-\epsilon_{0}(N)\right],
$$

where $C$ refers to the concurrence for two nearestneighbor qubits, and $\mathcal{E}_{0}$ is the ground-state energy. For a pair of qubits, the entanglement of formation can be obtained from the concurrence $C$

$$
E_{\mathrm{o} f}=h\left(\frac{1+\sqrt{1-C^{2}}}{2}\right),
$$

where $h(x)=-x \log _{2} x-(1-x) \log _{2}(1-x)$. The concurrence itself is a good measure of two-qubit entanglement, and we adopt it as our measure of pairwise entanglement.

For the case of finite temperature, the concurrence for two nearest qubits in the thermal state of the ring was found to be related to the thermodynamical function, the internal energy $U$ via [21, 22]

$$
C(N)=\max (0,-U / N),
$$

which connects the microscopic quantity, the concurrence, and the macroscopic quantity, the internal energy. Equations (1) and (3) show that the two-qubit entanglement and energies are closely related in the isotropic Heisenberg model.
Having known the relations between entanglement and energy for ground states and thermal states of the $X X X$ model, further questions arise that what are the entanglement properties of excited states, and what are the relationships between entanglement and excited-state energies. We will address the question in this paper. To study entanglement of excited states is not only interesting itself, but also help to understand the entanglement at finite temperatures. We also consider the entanglement versus energy in the quantum $X Y$ model, and show that the entanglement exhibits a symmetry, distinct from that in the $X X X$ model.

There exists another concept, the mixedness of a state, related to entanglement, is also central in quantum information theory [23]. For instance, Bose and Vedral have shown that entangled states become useless for quantum teleportation on exceeding a certain degree of mixedness [24]. For a pure bipartite state, the mixedness of one subsystem is equal to that of another one, and can be used to quantify bipartite entanglement. In this case, entanglement and mixedness are equivalent. We will study both entanglement and mixedness properties.

Entanglement and eigenenergy. We consider a physical model of a ring of $N$ qubits interacting via the isotropic Heisenberg $X X X$ Hamiltonian

$$
H=J \sum_{i=1}^{N-1} S_{i, i+1}+J S_{N, 1},
$$

where $S_{j, j+1}=\frac{1}{2}\left(1+\vec{\sigma}_{i} \cdot \vec{\sigma}_{i+1}\right)$ is the swap operator between qubit $i$ and $j, \vec{\sigma}_{i}=\left(\sigma_{i x}, \sigma_{i y}, \sigma_{i z}\right)$ is the vector of Pauli matrices, and $J$ is the exchange constant. Positive and negative $J$ correspond to the antiferromagnetic and ferromagnetic case, respectively. Note that we have assumed the periodic boundary condition.

When an energy level of our system is non-degenerate, the corresponding eigenstate is pure. When a $k$-th energy level is degenerate, we assume that the corresponding state is an equal mixture of all eigenstates with energy $\mathcal{E}_{k}$. Thus, the state correspoding to the $k$-th level with degeneracy becomes a mixed other than pure, keeping all symmetries of the Hamiltonian. A degenerate ground state 
is called thermal ground state in the sense that it can be obtained from the thermal state $\exp \left[-H /\left(k_{B} T\right)\right] / Z$ by taking the zero-temperature limit [2]. Here, $k_{B}$ is the Boltzman constant, $T$ the temperature, and $Z$ the partition function. The $k$-th eigenstate $\rho_{k}$ can be considered as the thermal ground state of the nonlinear Hamiltonian $H^{\prime}$ given by

$$
H^{\prime}=\left(H-\mathcal{E}_{k}\right)^{2} .
$$

Note that Hamiltonian $H^{\prime}$ inherits all symmetries of Hamiltonian $H$.

Due to the translational invariance of the system, all nearest-neighbour entanglements are identical. Thus, from now on, we consider the entanglement between qubit 1 and 2, representing nearest-neighbour pairwise entanglement. Another important $\mathrm{SU}(2)$ symmetry of the Hamiltonian guarantees that the reduced density ma$\operatorname{trix} \rho_{k}^{(12)}=\operatorname{Tr}_{3,4, \cdots, N}\left(\rho_{k}\right)$ for $k$-th eigenstate is given by

$$
\rho_{k}^{12}=\left(\begin{array}{llll}
u_{k+} & 0 & 0 & 0 \\
0 & u_{k-} & z_{k} & 0 \\
0 & z_{k} & u_{k-} & 0 \\
0 & 0 & 0 & u_{k+}
\end{array}\right)
$$

in the standard basis $\{|00\rangle,|01\rangle,|10\rangle,|11\rangle\}$, with

$$
u_{k \pm}=\left(1 \pm G_{k}^{z z}\right) / 4, z_{k}=G_{k}^{z z} / 2 .
$$

Here, $G_{k}^{\alpha \alpha}=\operatorname{Tr}\left(\sigma_{1 \alpha} \sigma_{2 \alpha} \rho_{k}\right), \alpha \in\{x, y, z\}$ are correlation functions which are equal due to the $\mathrm{SU}(2)$ symmetry.

From Hamiltonian $H$, a simple and useful relation between eigenenergy per site $\epsilon_{k}$ and the correlation function is obtained as

$$
\epsilon_{k}=\mathcal{E}_{k} / N=\frac{1+3 G_{k}^{z z}}{2} .
$$

Therefore, from Eqs. (6-8), the reduced density matrix is completely determined by the eigenenergy $\epsilon_{k}$. As all information about pairwise entanglement and mixedness of $\rho_{k}^{12}$ are contained in $\rho_{k}^{12}$, the eigenenergy completely determines the entanglement and mixedness. Next, we give a quantitative relation between the concurrence and the eigenenergy.

From the two-qubit reduced density matrix (6), the concurrence for two nearest qubits is obtained as [10]

$$
\begin{aligned}
C_{k}(N) & =2 \max \left(0,\left|z_{k}\right|-u_{k+}\right) \\
& =\max \left(0,\left|G_{k}^{z z}\right|-G_{k}^{z z} / 2-1 / 2\right) \\
& =\max \left(0,-3 G_{k}^{z z} / 2-1 / 2\right) \\
& =\max \left(0,-\epsilon_{k}\right) .
\end{aligned}
$$

The third equality follows from the inequality $\left|G_{k}^{z z}\right| \leq 1$, which is a special case of a more general result that $|\langle A\rangle| \leq 1$ for any Hermitian operator $A$ satisfying $A^{2}=1$. The last equality is obtained by using Eq. (8). Thus, we get a simple relation between the pairwise entanglement
TABLE I: The concurrences of the ground state, first and second excited states for the number of qubits from 3 to 16 .

\begin{tabular}{cccccccc}
$N$ & 3 & 4 & 5 & 6 & 7 & 8 & 9 \\
\hline$C_{0}$ & 0.0 & 0.5 & 0.2472 & 0.4343 & 0.3158 & 0.4128 & 0.3438 \\
\hline$C_{1}$ & 0.0 & 0.0 & 0.0 & 0.2060 & 0.0161 & 0.2821 & 0.1525 \\
\hline$C_{2}$ & - & 0.0 & 0.0 & 0.0 & 0.0055 & 0.1749 & 0.1342 \\
\hline$N$ & 10 & 11 & 12 & 13 & 14 & 15 & 16 \\
\hline$C_{0}$ & 0.4031 & 0.3580 & 0.3979 & 0.3661 & 0.3948 & 0.3712 & 0.3928 \\
\hline$C_{1}$ & 0.3184 & 0.2258 & 0.3386 & 0.2695 & 0.3509 & 0.2976 & 0.3590 \\
\hline$C_{2}$ & 0.2541 & 0.2100 & 0.2962 & 0.2570 & 0.3212 & 0.2877 & 0.3371
\end{tabular}

of two nearest qubits and the corresponding eigenenergy. A necessary condition for non-zero entanglement is that eigenenergy is negative, i.e., the negativity of eigenenergy implies pairwise entanglement. We also see that the ground state exhibits largest entanglement.

Now we apply the above result to investigate finite-size behaviours of the entanglement. Conformal invariance theory predicts the finite size behaviour of the ground state and the first excited state [25]

$$
\epsilon_{1}(N)-\epsilon_{0}(N) \approx \pi^{2} b / N^{2}
$$

with $b$ being identified with the central charge. From the above equation and Eq. (9), we immediately have

$$
C_{1}(N)-C_{0}(N) \approx-\pi^{2} b / N^{2},
$$

which is the finite size behaviour of the entanglement.

In Table I, we give the concurrences of the ground states, first and second excited states for the number of qubits from 3 to 16 . The ground state becomes entangled when $N \geq 4$ [21, 22]. However, the first (second) excited state becomes entangled when $N \geq 6(N \geq 7)$. The concurrence of the ground state decreases (increases) as even $N$ (odd $N$ ) increases. However, the concurrence of the first excited states increases as even $N$ or odd $N$ increases. This fact also holds for the second excited states. We see that the entanglement properties of the excited states are distinct from those of the ground states.

To clearly display behaviours of the pairwise entanglement corresponding to different energy levels, in Fig. 1, we plot the concurrences versus energy level index $k$ for different $N$. It is evident that for a fixed $N$ the concurrence decreases monotonically with the increase of $k$, and when $k$ is equal or larger than a threshold value $k_{\mathrm{th}}$, the entanglement vanishes. The threshold values $k_{\text {th }}$ is $N$-dependent, and the larger the number of qubits, the larger the threshold value. More and more excited states become entangled when the number of qubits increases.

Next, we study the mixedness properties of all eigenstates. The mixedness of a state $\varrho$ can be quantified by the linear entropy given by

$$
E=1-\operatorname{Tr}\left(\varrho^{2}\right) .
$$




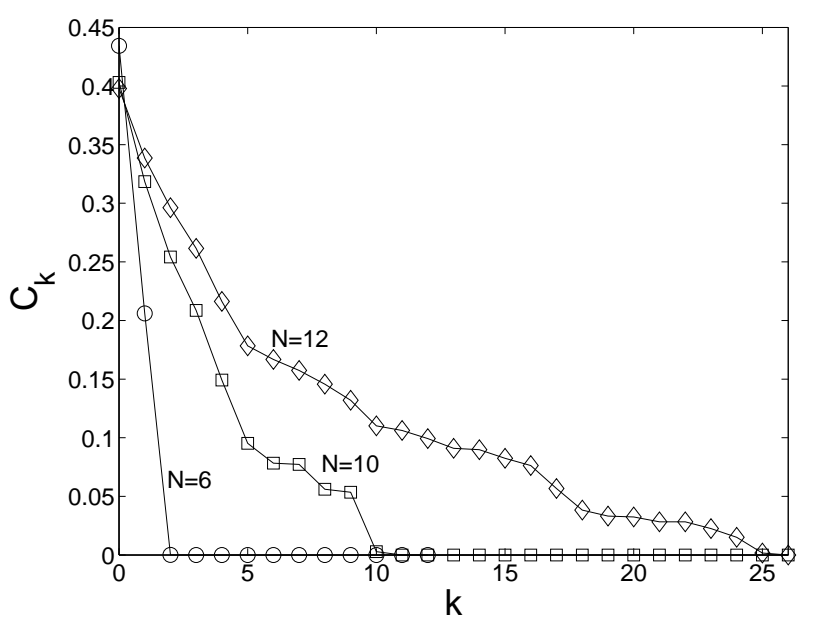

FIG. 1: The concurrence versus energy level index $k$ for different number of qubits.
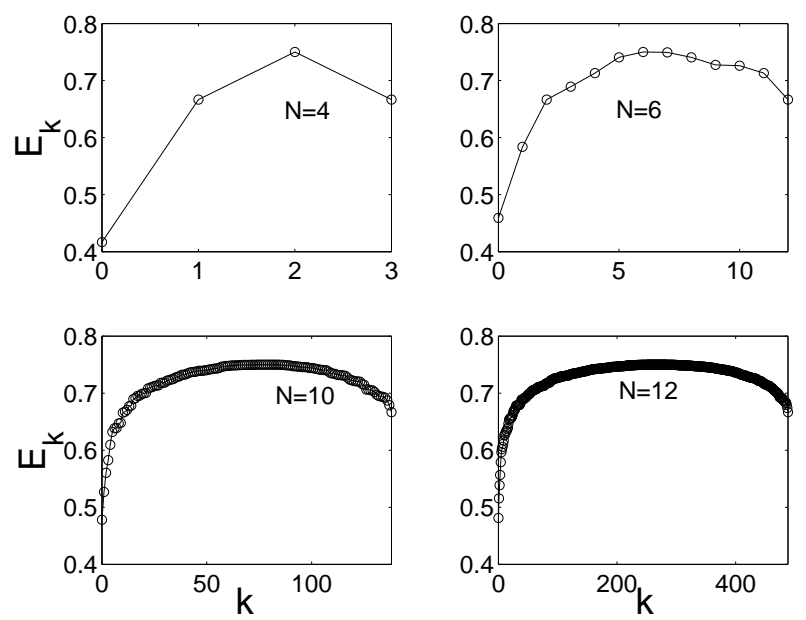

FIG. 2: The linear entropy versus $k$ for different $N$.

Then, from Eqs. (6), (7), (8), and (12), the linear entropy of the two-qubit state $\rho_{k}^{12}$ is obtained as

$$
E_{k}(N)=1-\frac{1}{3}\left[\epsilon_{k}^{2}(N)-\epsilon_{k}(N)+1\right],
$$

which is determined solely by the eigenenergy $\epsilon_{k}$ per site. Obviously, the linear entropy takes its maximum $3 / 4$ when $\epsilon_{k}=1 / 2$.

In Fig. 2, we plot the linear entropy versus $k$ for different number of qubits. As $k$ increases, the linear entropy monotonically increases and reaches a maximum, then monotonically decreases. In contrast to the concurrence, the linear entropy takes its maximum in the middle of energy levels, other than at the ground-state level. For instance, when $N=6$, there are 13 levels and the linear entropy takes its maximum when $k=6$. There are some eigenstates which are non-degenerate, and thus pure. In this case, the entanglement of between the two qubits and the rest quantified by the linear entropy is equivalent to
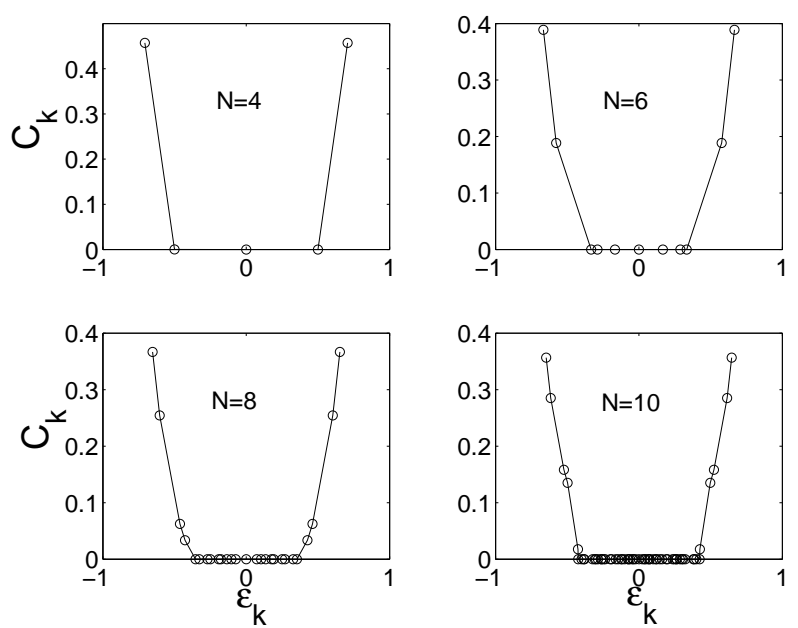

FIG. 3: The concurrence versus eigenenergy per site $\epsilon_{k}$ for different number of qubits in the $X Y$ model.

the mixedness of the two-qubit state. For instance, when $N=6$, the ground state, the second excited state, and the 10-th excited state are non-degenerate.

In the isotropic Heisenberg model described above, both entanglement and mixedness of an eigenstate are completely determined by the corresponding eigenenergy. This result is due to the many symmetries in the model. For other models, the relation between entanglement and energy may become more complicated. We now consider another well-established model, i.e., the Heisenberg $X Y$ model. The $X Y$ Hamiltonian is written as

$$
H_{\mathrm{XY}}=\frac{J}{2} \sum_{i=1}^{N}\left(\sigma_{i x} \otimes \sigma_{i+1 x}+\sigma_{i y} \otimes \sigma_{i+1 y}\right),
$$

where the periodic boundary condition is assumed again.

An interesting feature of this system is that for even $N$ if $\mathcal{E}$ is an eigenenergy, $-\mathcal{E}$ is an eigenenergy too. Thus, the eigenspectrum exhibits a symmetry, which is different from that in the $X X X$ model. The reason is that for even $N$ Hamiltonian $H_{\mathrm{XY}}$ anticommutes with the following operator

$$
\Lambda_{z}=\sigma_{1 z} \otimes \sigma_{3 z} \otimes \cdots \otimes \sigma_{N-1 z} .
$$

It is natural to ask if the entanglement and mixedness exhibit a similar symmetry. The answer is yes as we will see below.

By exact diagonalization method, we compute the concurrence and mixedness numerically in the $X Y$ model. In Fig. 3, we plot the concurrences for two nearest qubits versus eigenenergy per site $\epsilon_{k}$. It is evident that the concurrence shows a symmetry with respect to the point of $\epsilon_{k}=0$, which arises due to the fact that the operator $\Lambda_{z}$ is a local unitary operator, and the entanglement properties of the eigenstate with energy $\mathcal{E}$ are the same as those of the eigenstate with energy $-\mathcal{E}$. If we restrict ourselves 

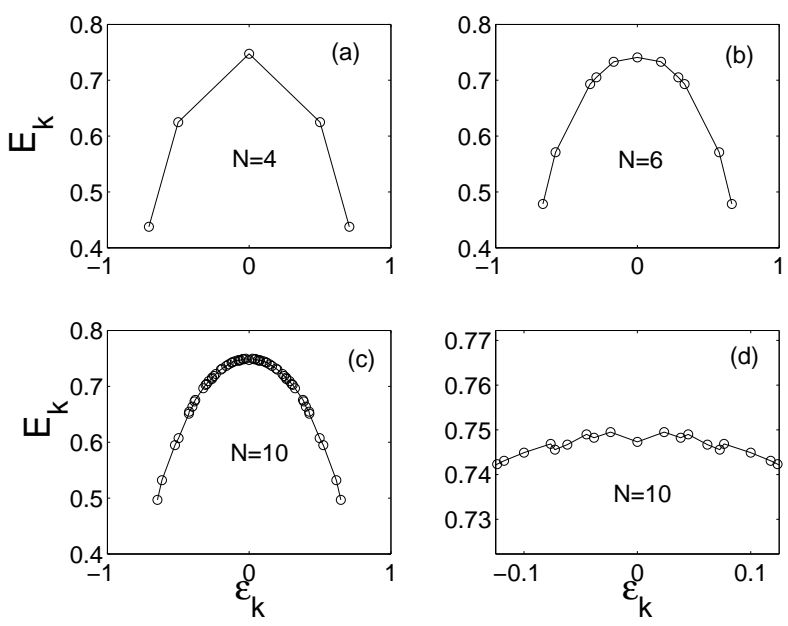

FIG. 4: The linear entropy versus eigenenergy per site $\epsilon_{k}$ for different $N$ in the $X Y$ model.

to the half part of the eigenspectrum $\left(\epsilon_{k} \leq 0\right)$, the behaviour of the concurrence is similar to that in the $X X X$ model, i.e., the concurrence monotonically decreases as $k$ increases and there also exist threshold values $k_{\mathrm{th}}$.

The linear entropy quantifying the mixedness of the two-qubit states is plotted in Fig. 4. It also exhibits a symmetry with respect to $\epsilon_{k}=0$. And it seems that the linear entropy takes its maximum at $\epsilon_{k}=0$ for any $N$ from Fig.4 (a-c). However, the partly enlarged version (Fig. 4 (d)) of Fig. 4 (c) clearly shows that the maximum is not exactly at $\epsilon_{k}=0$ for $N=10$. So, we see that the mixedness reaches its maximum at or near the point of $\epsilon_{k}=0$.

In conclusion, we have studied the entanglement and mixedness of all eigenstates in two well-established quantum spin models, i.e., the $X X X$ and $X Y$ models. For the $X X X$ model, the entanglement and mixedness of a pair of nearest-neighbor qubits in a eigenstate are completely determined by the corresponding eigenenergy. The entanglement decreases as we go from ground state to excited states, i.e. the more excited the system, the less the entanglement. The negativity of eigenenergy implies pairwise entanglement, i.e., the entanglement vanishes when the eigenenergy is large than zero. In contract to the entanglement property, the mixedness exhibits a maximum in the middle of energyspectrum, other than on the border. From the relation between entanglement and eigenenergy, we obtain the finite-size behaviors of the entanglement.

The properties of entanglement and mixedness in the $X Y$ model are distinct from those in the $X X X$ model. For even number of qubits, they both exhibits a symmetry with respect to the zero eigenenergy. However, for odd number of qubits, the symmetry breaks. It will be interesting to consider entanglement of all eigenstates in other physical systems such as quantum chaos system. The study of eigenvalue statistics and eigenvectors statistics naturally motivate us to study entanglement statistics, which is under consideration.

[1] C. H. Bennett, G. Brassard, C. Crepeau, R. Jozsa, A. Peres, and W. K. Wootters, Phys. Rev. Lett. 70, 1895 (1993).

[2] T. J. Osborne and M. A. Nielsen, Phys. Rev. A 66, 032110 (2002).

[3] M. A. Nielsen, Ph. D thesis, University of Mexico, 1998, quant-ph/0011036;

[4] M. C. Arnesen, S. Bose, and V. Vedral, Phys. Rev. Lett. 87, 017901 (2001).

[5] D. Gunlycke, V. M. Kendon, V. Vedral, and S. Bose, Phys. Rev. A 64, 042302 (2001).

[6] X. Wang, Phys. Rev. A 64, 012313 (2001); Phys. Lett. A 281, 101 (2001).

[7] X. Wang, H. Fu, and A. I. Solomon, J. Phys. A: Math. Gen. 34, 11307(2001); X. Wang and K. Mølmer, Eur. Phys. J. D 18, 385(2002).

[8] B. Q. Jin and V. E. Korepin, Phys. Rev. A 69, 062314 (2004)

[9] G. L. Kamta and A. F. Starace, Phys. Rev. Lett. 88, 107901 (2002).

[10] K. M. O'Connor and W. K. Wootters, Phys. Rev. A 63, 0520302 (2001).

[11] D. A. Meyer and N. R. Wallach, quant-ph/0108104.

[12] G. K. Brennen and S. S. Bullock, quant-ph/0406064.

[13] A. Wong and N. Christensen, Phys. Rev. A 63, 044301 (2001).

[14] A. Osterloh, L. Amico, G. Falci and R. Fazio, Nature 416, 608 (2002).

[15] Y. Sun, Y. G. Chen, and H. Chen, Phys. Rev. A 68, 044301 (2003).

[16] J. Vidal, G. Palacios, and R. Mosseri, Phys. Rev. A 69, 022107 (2004).

[17] N. Lambert, C. Emary, and T. Brandes, Phys. Rev. Lett. 92, 073602 (2004).

[18] G. Vidal, J. I. Latorre, E. Rico, and A. Kitaev, Phys. Rev. Lett. 90, 227902 (2003)

[19] S. J. Gu, H. Q. Lin, and Y. Q. Li, Phys. Rev. A 68, 042330 (2003).

[20] W. K. Wootters, Phys. Rev. Lett. 80, 2245 (1998).

[21] X. Wang and P. Zanardi, Phys. Lett. A 301, 1 (2002).

[22] X. Wang, Phys. Rev. A 66, 044305 (2002).

[23] G. Jaeger, A. V. Sergienko, B. E. A. Saleh, and M. C. Teich, Phys. Rev. A 68, 022318 (2003).

[24] S. Bose and V. Vedral, Phys. Rev. A 61, 040101 (2000).

[25] L. Cardy, in Phase transitions and Critical Phenomena, edited by C. Domb and J. L. Lebowitz (Academic, London, 1987), Vol. 11. 